\title{
Ilexpublesnins C-M, Eleven New Triterpene Saponins from the Roots of Ilex pubescens
}

Authors

Affiliations
Yuan Zhou ${ }^{1}$, Xing-Yun Chai ${ }^{2}$, Ke-Wu Zeng ${ }^{1}$, Jia-Yu Zhang ${ }^{1}$, Ning Li ${ }^{1}$, Yong Jiang ${ }^{1}$, Peng-Fei Tu ${ }^{1,2}$

${ }^{1}$ State Key Laboratory of Natural and Biomimetic Drugs, School of Pharmaceutical Sciences, Peking University Health Science Center, Beijing, China

${ }^{2}$ Modern Research Center for Traditional Chinese Medicine, Beijing University of Chinese Medicine, Beijing, China
Key words

- llex pubescens

- Aquifoliaceae

- ilexpublesnins C-M

- triterpene saponin

- 24-aldehyde group

- cytotoxicity received April 20, 2012

revised Sept. 2, 2012

accepted October 11, 2012

\section{Bibliography}

Dol http://dx.doi.org/

10.1055/s-0032-1327927

Published online November 16, 2012

Planta Med 2013; 79: 70-77

(c) Georg Thieme Verlag KG

Stuttgart · New York .

ISSN 0032-0943

\section{Correspondence}

Prof. Dr. Peng-Fei Tu

State Key Laboratory of Natural and Biomimetic Drugs

School of Pharmaceutical

Sciences

Peking University Health

Science Center

No. 38 Xue Yuan Road

Haidian District

Beijing 100191

China

Phone: + 861082802750

Fax: + 861082802750

pengfeitu@vip.163.com

\section{Abstract \\ $\nabla$}

Eleven new triterpene saponins, ilexpublesnins C-M (1-11), along with ten known analogues were isolated from the roots of Ilex pubescens. Their structures were elucidated on the basis of extensive spectroscopic analysis, including 1D and 2D NMR experiments. Compounds 1, 2, 10, and 11 contain a 24-aldehyde, which is rare for triterpene saponins from Ilex. These compounds

\section{Introduction}

$\nabla$

Ilex pubescens Hook. et Arn., known under the Chinese name 'Mao-dong-qing', is an evergreen shrub belonging to the Aquifoliaceae family. Its roots are commonly used as Chinese herbal medicine for treatment of cardiovascular disease and hypercholestemia in south China [1]. Previous phytochemical investigation on its roots and leaves led to the isolation of triterpene saponins [2], lignan glycosides [3], phenylethanols [4,5], and other minor compounds [6-8]. Pharmacological evaluation proved that Ilex pubescens extracts exhibited a variety of bioactivities, i.e., to enlarge blood vessels, improve microcirculation, ease blood pressure, inhibit platelet aggregation, prevent thrombosis, and reduce cardiac ischemia, as well as to decrease the excitation of the cardiac conduction system and other effects [9].

As part of a systematic research on seeking the bioactive constituents from Ilex species [10-18], a phytochemical investigation on the roots of $I$. pubescens was undertaken, which led to the isolation of eleven new triterpene saponins, namely ilexpublesnins C-M (1-11) ( $\mathbf{~ F i g . ~ 1 ) , ~ a n d ~ t e n ~}$ known ones (12-21). This paper describes the isolation and structural elucidation of these compounds. In addition, the cytotoxic evaluation for these compounds against the human cancer cell were evaluated in vitro for their cytotoxic effects on human cancer cell lines HepG2, HLE, BEL7402, BEL7403, BEL7405, MCF-7, and HeLa. Among them, only compounds 6 and 19 showed cytotoxicity against the MCF-7 cell line [inhibition (\%): 33.14 and 34.03, respectively].

Supporting information available online at http://www.thieme-connect.de/ejournals/toc/ plantamedica

lines HepG2, HLE, BEL-7402, BEL-7403, BEL7405, MCF-7, and HeLa is included.

\section{Materials and Methods}

$\nabla$

Instruments

HRESIMS were measured on a Bruker APEX IV FT_MS (7.0T) spectrometer in the positive ion mode. Optical rotations were measured on an Autopol-IV polarimeter (RudoPH Research analytical). IR spectra were obtained using a NEXUS-470 FTIR (Nicolet) spectrometer. 1D and 2D NMR spectra were recorded on Bruker Avance DRX400 and Vnmrs-500 spectrometers. Semipreparative HPLC was performed on a Waters model 2487 (Agilent ODS column, $250 \times 10 \mathrm{~mm}$ i.d., $5 \mu \mathrm{m}$ ) with an Alltech evaporative light scattering detector (ELSD). GC analysis was carried out on an Agilent $6890 \mathrm{~N}$ gas chromatograph, with an HP-5 capillary column ( $28 \mathrm{~m} \times 0.32 \mathrm{~mm})$ and an FID detector operated at $260^{\circ} \mathrm{C}$ (column temp. $180^{\circ} \mathrm{C}$ ), and $1.0 \mathrm{~mL} / \mathrm{min}_{2}$ as the carrier gas. Macroporous resin (HPD100) was purchased from Hebei Bao-En Biotech Ltd. Thin-layer and column chromatography was performed using silica gel (Qingdao Haiyang Chemical Co. Ltd., $\mathrm{GF}_{254}$ or 200-300 mesh), Sephadex LH-20 (Pharmacia Biotech Ltd.), and ODS (Merck \& Co., Inc.). All the solvents were 
of analytical grade and purchased from Beijing Chemical Company Ltd.

\section{Plant material}

The roots of Ilex pubescens were purchased from Guilin San-Jin Pharmaceutical Co. Ltd and originally collected from Guangxi province, China. The plants were identified by Prof. Peng-Fei Tu (one of the authors in this paper). A voucher specimen (No. 091005) is deposited in the Herbarium of Modern Research Center for Traditional Chinese Medicine (TCM), Peking University, Beijing.

\section{Extraction and isolation}

Dry crude materials $(18 \mathrm{~kg}$ ) were grinded and extracted with 70\% $\mathrm{EtOH}$ at the temperature of $70^{\circ} \mathrm{C}$. After the retrieval of the etha$\mathrm{nol}$, the residue suspended in water $(50 \mathrm{~L})$ was subjected to column chromatograph (CC) on macroporous resin with an EtOH- $\mathrm{H}_{2} \mathrm{O}$ gradient $(30: 70,70: 30,90: 10)$ to yield three fractions (Frs.1-3). Fr.2 (160 g) was chromatographed on silica gel $(2.5 \mathrm{~kg}, 100 \times 10 \mathrm{~cm})$ with a gradient of $\mathrm{CHCl}_{3}-\mathrm{MeOH}(40: 1-$ $1: 1$ ) elution to yield thirteen fractions (Frs. A-M). Then these fractions were applied to CC on silica gel, Sephadex LH-20, ODS, and semipreparative HPLC to afford compounds $1(30 \mathrm{mg}), 2$ (5 mg), 3 (80 mg), 4 (200 mg), 5 (15 mg), 6 (15 mg), 7 (50 mg), 8 (9 mg), 9 (12 mg), 10 (28 mg), 11 (10 mg), 12 (9 mg), 13 (120 mg), 14 (160 mg), 15 (250 mg), 16 (10 mg), 17 (8 mg), 18 (25 mg), 19 $(24 \mathrm{mg}), \mathbf{2 0}(22 \mathrm{mg})$, and 21 (500 mg). For detailed isolation and purification protocol, see Supporting Information.

Ilexpublesnin $C(\mathbf{1})$ : white, amorphous powder; $[\alpha]_{D}^{20}+9.47(c$ 0.19, MeOH); IR (KBr) $v_{\max } 3423,2933,1719,1611,1072 \mathrm{~cm}^{-1}$; ${ }^{1} \mathrm{H}$ and ${ }^{13} \mathrm{C}$ NMR data (pyridine- $d_{5}, 500 / 125 \mathrm{MHz}$ ), see 0 Tables 1 and 2; HRESIMS $\mathrm{m} / z$ 842.45015 $\left[\mathrm{M}+\mathrm{NH}_{4}\right]^{+}$(calcd. for $\left.\mathrm{C}_{42} \mathrm{H}_{64} \mathrm{O}_{16} \mathrm{NH}_{4}, 842.45326\right)$.

Ilexpublesnin $D\left(\right.$ 2): white, amorphous powder; $[\alpha]_{D}^{20}+9.34(c$ 0.15, MeOH); IR (KBr) $v_{\max } 3433,2932,1692,1631,1068 \mathrm{~cm}^{-1}$; ${ }^{1} \mathrm{H}$ and ${ }^{13} \mathrm{C}$ NMR data (pyridine- $d_{5}, 500 / 125 \mathrm{MHz}$ ), see $\odot$ Tables 1 and 2; HRESIMS $\mathrm{m} / \mathrm{z} 680.40011\left[\mathrm{M}+\mathrm{NH}_{4}\right]^{+}$(calcd. for $\left.\mathrm{C}_{36} \mathrm{H}_{54} \mathrm{O}_{11} \mathrm{NH}_{4}, 680.40044\right)$.

Ilexpublesnin $E$ (3): white, amorphous powder; $[\alpha]_{D}^{20}-56.0(c$ 0.10, MeOH); IR (KBr) $v_{\max } 3420,2933,1750,1642,1073 \mathrm{~cm}^{-1}$; ${ }^{1} \mathrm{H}$ and ${ }^{13} \mathrm{C}$ NMR data (pyridine- $d_{5}, 400 / 100 \mathrm{MHz}$ ), see $\odot$ Tables 1 and 2; HRESIMS $\mathrm{m} / \mathrm{z} 1092.59670\left[\mathrm{M}+\mathrm{NH}_{4}\right]^{+}$(calcd. for $\mathrm{C}_{53} \mathrm{H}_{86} \mathrm{O}_{22} \mathrm{NH}_{4}, 1092.59490$ ).

Ilexpublesnin $F(4)$ : white, amorphous powder; $[\alpha]_{D}^{20}+52.6(c$ 0.15, MeOH); IR (KBr) $v_{\max } 3433,2932,1695,1078 \mathrm{~cm}^{-1} ;{ }^{1} \mathrm{H}$ and ${ }^{13} \mathrm{C}$ NMR data (pyridine- $d_{5}, 400 / 100 \mathrm{MHz}$ ), see $\boldsymbol{O}$ Tables 1 and 2; HRESIMS $m / z 766.47571\left[\mathrm{M}+\mathrm{NH}_{4}\right]^{+}$(calcd. for $\mathrm{C}_{41} \mathrm{H}_{64} \mathrm{O}_{12} \mathrm{NH}_{4}$, 766.47360).

Ilexpublesnin $G(5)$ : white, amorphous powder; $[\alpha]_{D}^{20}+3.3(c 0.12$, $\mathrm{MeOH}) ; \mathrm{IR}(\mathrm{KBr}) v_{\max } 3420,2936,1731,1072 \mathrm{~cm}^{-1} ;{ }^{1} \mathrm{H}$ and ${ }^{13} \mathrm{C}$ NMR data (pyridine- $d_{5}, 500 / 125 \mathrm{MHz}$ ), see 0 Tables 1 and 2; HRESIMS $\mathrm{m} / \mathrm{z} 1565.89765[2 \mathrm{M}+\mathrm{H}]^{+}$(calcd. for $\mathrm{C}_{82} \mathrm{H}_{133} \mathrm{O}_{28}$, 1565.89779).

Ilexpublesnin $H(6)$ : white, amorphous powder; $[\alpha]_{D}^{20}+8.33(c$ 0.12, MeOH); IR (KBr) $v_{\max } 3434,2943,2879,1732,1639$, $1073 \mathrm{~cm}^{-1} ;{ }^{1} \mathrm{H}$ and ${ }^{13} \mathrm{C}$ NMR data (pyridine- $d_{5}, 400 / 100 \mathrm{MHz}$ ),

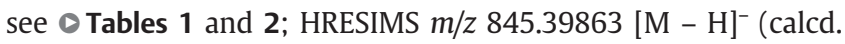
for $\mathrm{C}_{41} \mathrm{H}_{66} \mathrm{O}_{16} \mathrm{~S}, 845.39988$ ).

Ilexpublesnin $I(7)$ : white, amorphous powder; $[\alpha]_{\mathrm{D}}^{20}+2.33$ ( c 0.15 , $\mathrm{MeOH}) ; \mathrm{IR}(\mathrm{KBr}) v_{\max } 3453,2940,2875,1738,1637,1063 \mathrm{~cm}^{-1}$; ${ }^{1} \mathrm{H}$ and ${ }^{13} \mathrm{C}$ NMR data (pyridine- $d_{5}, 400 / 100 \mathrm{MHz}$ ), see 0 Tables 1 and 2; HRESIMS $m / z 869.39648[\mathrm{M}+\mathrm{Na}]^{+}$(calcd. for $\mathrm{C}_{41} \mathrm{H}_{66} \mathrm{O}_{16} \mathrm{~S}$ $\mathrm{Na}, 869.39638)$.

Ilexpublesnin $J(8)$ : white, amorphous powder; $[\alpha]_{\mathrm{D}}^{20}+2.0$ (c 0.08 , $\mathrm{MeOH}) ; \mathrm{IR}(\mathrm{KBr}) v_{\max } 3428,2930,2879,1730,1076 \mathrm{~cm}^{-1}$; ${ }^{1} \mathrm{H}$ and ${ }^{13} \mathrm{C}$ NMR data (pyridine- $d_{5}, 400 / 100 \mathrm{MHz}$ ), see $\bigcirc$ Tables 1 and 2; HRESIMS $\mathrm{m} / z$ 1108.58861 $\left[\mathrm{M}+\mathrm{NH}_{4}\right]^{+}$(calcd. for $\mathrm{C}_{53} \mathrm{H}_{86} \mathrm{O}_{23} \mathrm{NH}_{4}$, 1108.58981).

Ilexpublesnin $K(9)$ : white, amorphous powder; $[\alpha]_{D}^{20}-4.1$ ( $c 0.10$, $\mathrm{MeOH}$ ); IR (KBr) $v_{\max } 3421,29292875,1732,1075 \mathrm{~cm}^{-1}$; ${ }^{1} \mathrm{H}$ and ${ }^{13} \mathrm{C}$ NMR data (pyridine- $d_{5}, 400 / 100 \mathrm{MHz}$ ), see $\odot$ Tables 1 and 2; HRESIMS $m / z$ 1078.58298 $\left[\mathrm{M}+\mathrm{NH}_{4}\right]^{+}$(calcd. for $\mathrm{C}_{53} \mathrm{H}_{86} \mathrm{O}_{23} \mathrm{NH}_{4}$, 1078.57925).

Ilexpublesnin $L$ (10): white, amorphous powder; $[\alpha]_{\mathrm{D}}^{20}+12.05$ (c 0.19, MeOH); IR (KBr) $v_{\max } 3427,2941,1718,1615,1071 \mathrm{~cm}^{-1}$; ${ }^{1} \mathrm{H}$ and ${ }^{13} \mathrm{C}$ NMR data (pyridine- $d_{5}, 500 / 125 \mathrm{MHz}$ ), see $\odot$ Tables 1 and 2; HRESIMS $\mathrm{m} / z$ 842.45024 $\left[\mathrm{M}+\mathrm{NH}_{4}\right]^{+}$(calcd. for $\left.\mathrm{C}_{42} \mathrm{H}_{64} \mathrm{O}_{16} \mathrm{NH}_{4}, 842.45326\right)$.

Ilexpublesnin $M(11)$ : white, amorphous powder; $[\alpha]_{\mathrm{D}}^{20}+8.57$ (c 0.14, MeOH); IR (KBr) $v_{\max } 3433,2944,1689,1073 \mathrm{~cm}^{-1} ;{ }^{1} \mathrm{H}$ and ${ }^{13} \mathrm{C}$ NMR data (pyridine- $d_{5}, 500 / 125 \mathrm{MHz}$ ), see $\bigcirc$ Tables 1 and 2; HRESIMS $\mathrm{m} / \mathrm{z} 680.39823\left[\mathrm{M}+\mathrm{NH}_{4}\right]^{+}$(calcd. for $\mathrm{C}_{36} \mathrm{H}_{54} \mathrm{O}_{11} \mathrm{NH}_{4}$, 680.40044).

\section{Acid hydrolysis}

Compounds 1, 3-11 (4 mg), and $\mathbf{2}(2 \mathrm{mg})$ were heated in $3 \mathrm{~mL}$ of $10 \% \mathrm{HCl}$-dioxane $(1: 1)$ at $90^{\circ} \mathrm{C}$ for $4 \mathrm{~h}$ in a sealed amp. After the dioxane was removed, the solution was extracted with EtOAc $(3 \mathrm{~mL} \times 3)$ to yield the aglycon and the sugar, respectively. The sugar components in the aqueous layer left after acid hydrolysis of 1-11 were analyzed by silica gel TLC by comparison with standard sugars. The solvent system was n-BuOH-TEA- $\mathrm{H}_{2} \mathrm{O}$ ( $60: 0.7: 30)$, and spots were visualized by spraying with phenylamine-diphenylamine, and then heated at $200^{\circ} \mathrm{C}$ for $1 \mathrm{~min}$. For the sugars of 1-11, the $R_{f}$ of glucuronic acid, glucose, arabinose, xylose, and rhamnose by TLC was $0.44,0.64,0.65,0.71,0.77$, respectively. The results were confirmed by GC analysis of the methyl sugar peracetates. The aqueous layer was evaporated and dissolved in anhydrous pyridine $(100 \mu \mathrm{L}) ; 0.1 \mathrm{~mL}$ cysteine methyl ester hydrochloride $(200 \mu \mathrm{L})$ was added, and the mixture was warmed at $60^{\circ} \mathrm{C}$ for $1 \mathrm{~h}$. The trimethysilylation reagent HMDS-TMCS (hexamethyldisilazane-trimethylchlorosilanepyridine, 2:1:10) (Acros Organics) was added and warmed at $60^{\circ} \mathrm{C}$ for $30 \mathrm{~min}$. The thiazolidine derivatives were analyzed by GC for sugar identification. The retention times of L-arabinose $\left(t_{R}\right.$, $5.19 \mathrm{~min})$, L-rhamnose ( $\left.t_{R}, 5.34 \mathrm{~min}\right)$, D-xylose ( $\left.t_{\mathrm{R}}, 5.46 \mathrm{~min}\right)$, Dglucose $\left(t_{R}, 11.63 \mathrm{~min}\right)$, and $\mathrm{D}$-glucuronic acid $\left(t_{\mathrm{R}}, 23.56 \mathrm{~min}\right)$ were confirmed by comparison with those of authentic standards [10].

\section{Cytotoxicity assay}

The method described in the literature [19] was used for the measurement of cytotoxic activities against human cancer cell lines. For detailed protocols, see Supporting Information.

\section{Supporting information}

The detailed isolation and purification protocol of 1-11, cytotoxicity assay and MTT data of 1-21, structures of 12-21, and the HRESIMS, IR, 1D and 2D NMR spectra of 1-11 are available as Supporting Information. 
Table $1{ }^{1} \mathrm{H}$ NMR data (in pyridine- $d_{5}$ ) of 1, 2, 5, 10, 11 (500 MHz), 3, 4, and 6-9 (400 MHz), $J$ in $\mathrm{Hz}$ and $\delta$ in ppm.

\begin{tabular}{|c|c|c|c|c|c|c|c|c|c|c|c|}
\hline Pos. & 1 & 2 & 3 & 4 & 5 & 6 & 7 & 8 & 9 & 10 & 11 \\
\hline 1 & $\begin{array}{l}0.85 \mathrm{~m} \\
1.50 \mathrm{~m}\end{array}$ & $\begin{array}{l}0.87 \mathrm{~m} \\
1.45 \mathrm{~m}\end{array}$ & $\begin{array}{l}0.94 \mathrm{~m} \\
1.52 \mathrm{~m}\end{array}$ & $\begin{array}{l}0.84 \mathrm{~m} \\
1.54 \mathrm{~m}\end{array}$ & $\begin{array}{l}0.89 \mathrm{~m} ; \\
1.53 \mathrm{~m}\end{array}$ & $\begin{array}{l}0.87 \mathrm{~m} \\
1.52 \mathrm{~m}\end{array}$ & $\begin{array}{l}0.89 \mathrm{~m} \\
1.52 \mathrm{~m}\end{array}$ & $\begin{array}{l}0.88 \mathrm{~m} \\
1.52 \mathrm{~m}\end{array}$ & $\begin{array}{l}0.89 \mathrm{~m} \\
1.53 \mathrm{~m}\end{array}$ & $\begin{array}{l}0.81 \mathrm{~m} \\
1.44 \mathrm{~m}\end{array}$ & $\begin{array}{l}0.82 \mathrm{~m} \\
1.43 \mathrm{~m}\end{array}$ \\
\hline 2 & $\begin{array}{l}2.00 \mathrm{~m} \\
2.46 \mathrm{~m}\end{array}$ & $\begin{array}{l}2.02 \mathrm{~m} \\
2.44 \mathrm{~m}\end{array}$ & $\begin{array}{l}1.86 \mathrm{~m} \\
2.08 \mathrm{~m}\end{array}$ & $\begin{array}{l}1.87 \mathrm{~m} ; \\
2.06 \mathrm{~m}\end{array}$ & $\begin{array}{l}1.99 \mathrm{~m} \\
2.23 \mathrm{~m}\end{array}$ & $\begin{array}{l}1.82 \mathrm{~m} \\
2.02 \mathrm{~m}\end{array}$ & $\begin{array}{l}1.90 \mathrm{~m} ; \\
2.07\end{array}$ & $\begin{array}{l}1.78 \mathrm{~m} \\
2.06 \mathrm{~m}\end{array}$ & $\begin{array}{l}1.77 \mathrm{~m} \\
2.08 \mathrm{~m}\end{array}$ & $\begin{array}{l}2.09 \mathrm{~m} \\
2.48 \mathrm{~m}\end{array}$ & $\begin{array}{l}2.10 \mathrm{~m} \\
2.44 \mathrm{~m}\end{array}$ \\
\hline 3 & $3.56 \mathrm{~m}$ & $3.59 \mathrm{~m}$ & $3.24 \mathrm{~m}$ & $3.27 \mathrm{~m}$ & $3.52 \mathrm{~m}$ & $3.29 \mathrm{~m}$ & $3.24 \mathrm{~m}$ & $3.21 \mathrm{~m}$ & $3.24 \mathrm{~m}$ & $3.55 \mathrm{~m}$ & $3.57 \mathrm{~m}$ \\
\hline 5 & $1.05 \mathrm{~m}$ & $1.06 \mathrm{~m}$ & $\begin{array}{l}0.83 d \\
(11.6)\end{array}$ & $\begin{array}{l}0.72 \mathrm{~d} \\
(11.6)\end{array}$ & $0.93 \mathrm{~m}$ & $\begin{array}{l}0.76 \mathrm{~d} \\
(11.6)\end{array}$ & $0.79 \mathrm{~m}$ & $\begin{array}{l}0.76 \mathrm{~d} \\
(11.6)\end{array}$ & $\begin{array}{l}0.79 d \\
(11.6)\end{array}$ & $1.00 \mathrm{~m}$ & $1.04 \mathrm{~m}$ \\
\hline 6 & $\begin{array}{l}1.37 \mathrm{~m} \\
1.74 \mathrm{~m}\end{array}$ & $\begin{array}{l}1.29 \mathrm{~m} \\
1.48 \mathrm{~m}\end{array}$ & $\begin{array}{l}1.31 \mathrm{~m} \\
1.45 \mathrm{~m}\end{array}$ & $\begin{array}{l}1.23 \mathrm{~m} \\
1.45 \mathrm{~m}\end{array}$ & $\begin{array}{l}1.33 \mathrm{~m} ; \\
1.62 \mathrm{~m}\end{array}$ & $\begin{array}{l}1.27 \mathrm{~m} \\
1.46 \mathrm{~m}\end{array}$ & $\begin{array}{l}1.26 \mathrm{~m} \\
1.45 \mathrm{~m}\end{array}$ & $\begin{array}{l}1.28 \mathrm{~m} \\
1.45 \mathrm{~m}\end{array}$ & $\begin{array}{l}1.27 \mathrm{~m} \\
1.45 \mathrm{~m}\end{array}$ & $\begin{array}{l}1.41 \mathrm{~m} \\
1.75 \mathrm{~m}\end{array}$ & $\begin{array}{l}1.42 \mathrm{~m} \\
1.80 \mathrm{~m}\end{array}$ \\
\hline 7 & $\begin{array}{l}1.38 \mathrm{~m} \\
1.47 \mathrm{~m}\end{array}$ & $\begin{array}{l}1.32 \mathrm{~m} \\
1.48 \mathrm{~m}\end{array}$ & $\begin{array}{l}1.41 \mathrm{~m} ; \\
1.59 \mathrm{~m}\end{array}$ & $\begin{array}{l}1.52 \mathrm{~m} \\
2.41 \mathrm{~m}\end{array}$ & $\begin{array}{l}1.35 \mathrm{~m} \\
1.50 \mathrm{~m}\end{array}$ & $\begin{array}{l}1.30 \mathrm{~m} \\
1.58 \mathrm{~m}\end{array}$ & $\begin{array}{l}1.38 \mathrm{~m} ; \\
1.52 \mathrm{~m}\end{array}$ & $\begin{array}{l}1.38 \mathrm{~m} \\
1.45 \mathrm{~m}\end{array}$ & $\begin{array}{l}1.38 ; \\
1.45 \mathrm{~m}\end{array}$ & $\begin{array}{l}1.33 \mathrm{~m} \\
1.48 \mathrm{~m}\end{array}$ & $\begin{array}{l}1.28 \mathrm{~m} \\
1.43 \mathrm{~m}\end{array}$ \\
\hline 9 & $1.72 \mathrm{~m}$ & $1.79 \mathrm{~m}$ & $1.78 \mathrm{~m}$ & $1.46 \mathrm{~m}$ & $1.78 \mathrm{~m}$ & $1.80 \mathrm{~m}$ & $1.76 \mathrm{~m}$ & $1.78 \mathrm{~m}$ & $1.78 \mathrm{~m}$ & $1.74 \mathrm{~m}$ & $1.81 \mathrm{~m}$ \\
\hline 11 & $\begin{array}{l}1.97 \mathrm{~m} \\
2.05 \mathrm{~m}\end{array}$ & $\begin{array}{l}1.86 \mathrm{~m} \\
2.04 \mathrm{~m}\end{array}$ & $\begin{array}{l}1.68 \mathrm{~m} \\
2.00 \mathrm{~m}\end{array}$ & $\begin{array}{l}1.61 \mathrm{~m} ; \\
1.91 \mathrm{~m}\end{array}$ & $\begin{array}{l}1.62 \mathrm{~m} \\
1.98 \mathrm{~m}\end{array}$ & $\begin{array}{l}1.68 \mathrm{~m} \\
2.07 \mathrm{~m}\end{array}$ & $\begin{array}{l}1.62 \mathrm{~m} \\
2.00 \mathrm{~m}\end{array}$ & $\begin{array}{l}1.61 \mathrm{~m} \\
2.01 \mathrm{~m}\end{array}$ & $\begin{array}{l}1.62 \mathrm{~m} \\
2.00 \mathrm{~m}\end{array}$ & $\begin{array}{l}1.85 \mathrm{~m} \\
2.06 \mathrm{~m}\end{array}$ & $\begin{array}{l}1.88 \mathrm{~m} \\
1.96 \mathrm{~m}\end{array}$ \\
\hline 12 & $5.49 \mathrm{brs}$ & $5.56 \mathrm{brs}$ & $5.52 \mathrm{brs}$ & $5.60 \mathrm{t}(4.0)$ & $5.48 \mathrm{brs}$ & $5.52 \mathrm{brs}$ & $5.48 \mathrm{brs}$ & $5.49 \mathrm{brs}$ & $5.48 \mathrm{brs}$ & $5.44 \mathrm{brs}$ & $5.51 \mathrm{brs}$ \\
\hline 15 & $\begin{array}{l}1.16 \mathrm{~m} \\
2.37 \mathrm{~m}\end{array}$ & $\begin{array}{l}1.25 \mathrm{~m} \\
1.99 \mathrm{~m}\end{array}$ & $\begin{array}{l}1.13 \mathrm{~m} \\
2.42 \mathrm{~m}\end{array}$ & $\begin{array}{l}2.13 \mathrm{~m} \\
2.30 \mathrm{~m}\end{array}$ & $\begin{array}{l}1.26 \mathrm{~m} \\
1.93 \mathrm{~m}\end{array}$ & $\begin{array}{l}1.25 \mathrm{~m} \\
2.48 \mathrm{~m}\end{array}$ & $\begin{array}{l}1.25 \mathrm{~m} \\
2.45 \mathrm{~m}\end{array}$ & $\begin{array}{l}1.22 \mathrm{~m} \\
2.44 \mathrm{~m}\end{array}$ & $\begin{array}{l}1.22 \mathrm{~m} \\
2.48 \mathrm{~m}\end{array}$ & $\begin{array}{l}1.21 \mathrm{~m} \\
2.25 \mathrm{~m}\end{array}$ & $\begin{array}{l}1.21 \mathrm{~m} \\
2.09 \mathrm{~m}\end{array}$ \\
\hline 16 & $\begin{array}{l}1.73 \mathrm{~m} \\
1.96 \mathrm{~m}\end{array}$ & $\begin{array}{l}1.24 \mathrm{~m} \\
2.25 \mathrm{~m}\end{array}$ & $\begin{array}{l}1.86 \mathrm{~m} \\
2.06 \mathrm{~m}\end{array}$ & $\begin{array}{l}2.30 \mathrm{~m} \\
2.43 \mathrm{~m}\end{array}$ & $\begin{array}{l}2.04 \mathrm{~m} \\
2.11 \mathrm{~m}\end{array}$ & $\begin{array}{l}1.82 \mathrm{~m} \\
2.07 \mathrm{~m}\end{array}$ & $\begin{array}{l}1.87 \mathrm{~m} ; \\
2.07 \mathrm{~m}\end{array}$ & $\begin{array}{l}1.93 \mathrm{~m} ; \\
2.06 \mathrm{~m}\end{array}$ & $\begin{array}{l}1.91 \mathrm{~m} \\
2.09 \mathrm{~m}\end{array}$ & $\begin{array}{l}1.17 \mathrm{~m} ; \\
2.02 \mathrm{~m}\end{array}$ & $\begin{array}{l}1.16 \mathrm{~m} \\
2.07 \mathrm{~m}\end{array}$ \\
\hline 18 & $2.89 \mathrm{~s}$ & $3.02 \mathrm{~s}$ & $2.91 \mathrm{~s}$ & & $3.17 \mathrm{~s}$ & $3.18 \mathrm{brs}$ & $3.16 \mathrm{~s}$ & $3.17 \mathrm{~s}$ & $3.16 \mathrm{~s}$ & 3.46 br s & $3.59 \mathrm{br} \mathrm{s}$ \\
\hline 19 & & & & & & & & & & 3.55 br s & 3.59 br s \\
\hline 20 & $1.32 \mathrm{~m}$ & $1.36 \mathrm{~m}$ & $1.33 \mathrm{~m}$ & $2.11 \mathrm{~m}$ & $2.00 \mathrm{~m}$ & $1.95 \mathrm{~m}$ & $1.93 \mathrm{~m}$ & $1.93 \mathrm{~m}$ & $1.91 \mathrm{~m}$ & & \\
\hline 21 & $\begin{array}{l}1.96 \mathrm{~m} \\
2.50 \mathrm{~m}\end{array}$ & $\begin{array}{l}1.44 \mathrm{~m} \\
2.44 \mathrm{~m}\end{array}$ & $\begin{array}{l}1.85 \mathrm{~m} \\
2.04 \mathrm{~m}\end{array}$ & $\begin{array}{l}1.55 \mathrm{~m} ; \\
1.66 \mathrm{~m}\end{array}$ & $\begin{array}{l}1.93 \mathrm{~m} \\
2.48 \mathrm{~m}\end{array}$ & $\begin{array}{l}1.68 \mathrm{~m} \\
2.00 \mathrm{~m}\end{array}$ & $\begin{array}{l}1.69 \mathrm{~m} ; \\
2.07 \mathrm{~m}\end{array}$ & $\begin{array}{l}1.69 \mathrm{~m} ; \\
1.99 \mathrm{~m}\end{array}$ & $\begin{array}{l}1.69 \mathrm{~m} ; \\
1.98 \mathrm{~m}\end{array}$ & $\begin{array}{l}1.23 \mathrm{~m} \\
1.38 \mathrm{~m}\end{array}$ & $\begin{array}{l}1.24 \mathrm{~m} \\
1.40 \mathrm{~m}\end{array}$ \\
\hline 22 & $\begin{array}{l}1.79 \mathrm{~m} \\
2.01 \mathrm{~m}\end{array}$ & $\begin{array}{l}1.79 \mathrm{~m} \\
2.08 \mathrm{~m}\end{array}$ & $\begin{array}{l}1.81 \mathrm{~m} \\
2.06 \mathrm{~m}\end{array}$ & $\begin{array}{l}\text { 2.29; } \\
2.41 \mathrm{~m}\end{array}$ & $\begin{array}{l}1.92 \mathrm{~m} \\
2.20 \mathrm{~m}\end{array}$ & $\begin{array}{l}1.87 \mathrm{~m} \\
2.07 \mathrm{~m}\end{array}$ & $\begin{array}{l}1.87 \mathrm{~m} ; \\
2.07 \mathrm{~m}\end{array}$ & $\begin{array}{l}1.88 \mathrm{~m} \\
2.09 \mathrm{~m}\end{array}$ & $\begin{array}{l}1.87 \mathrm{~m} \\
2.09 \mathrm{~m}\end{array}$ & $\begin{array}{l}1.91 \mathrm{~m} ; \\
2.00 \mathrm{~m}\end{array}$ & $\begin{array}{l}2.00 \mathrm{~m} \\
2.05 \mathrm{~m}\end{array}$ \\
\hline 23 & $1.54 \mathrm{~s}$ & $1.58 \mathrm{~s}$ & $1.31 \mathrm{~s}$ & $1.25 \mathrm{~s}$ & $1.51 \mathrm{~s}$ & $1.21 \mathrm{~s}$ & $1.37 \mathrm{~m}$ & $1.20 \mathrm{~s}$ & $1.37 \mathrm{~m}$ & $1.52 \mathrm{~s}$ & $1.59 \mathrm{~s}$ \\
\hline 24 & $10.32 \mathrm{~s}$ & $10.32 \mathrm{~s}$ & $1.06 \mathrm{~s}$ & $1.05 \mathrm{~s}$ & $\begin{array}{l}3.61 \mathrm{~d} \\
(11.5) \\
4.36 \mathrm{~d} \\
(11.5)\end{array}$ & $0.96 \mathrm{~s}$ & $1.15 \mathrm{~m}$ & $1.06 \mathrm{~s}$ & $1.15 \mathrm{~m}$ & $10.30 \mathrm{~s}$ & $10.32 \mathrm{~s}$ \\
\hline 25 & $0.72 \mathrm{~s}$ & $0.69 \mathrm{~s}$ & $0.86 \mathrm{~s}$ & $0.83 \mathrm{~s}$ & $0.84 \mathrm{~s}$ & $0.89 \mathrm{~s}$ & $0.86 \mathrm{~m}$ & $0.89 \mathrm{~s}$ & $0.89 \mathrm{~m}$ & $0.69 \mathrm{~s}$ & $0.69 \mathrm{~s}$ \\
\hline 26 & $1.06 \mathrm{~s}$ & $0.98 \mathrm{~s}$ & $1.16 \mathrm{~s}$ & $0.97 \mathrm{~s}$ & $1.16 \mathrm{~s}$ & $1.18 \mathrm{~s}$ & $1.14 \mathrm{~s}$ & $1.18 \mathrm{~s}$ & $1.18 \mathrm{~m}$ & $1.12 \mathrm{~s}$ & $0.93 \mathrm{~s}$ \\
\hline 27 & $1.65 \mathrm{~s}$ & $1.72 \mathrm{~s}$ & $1.67 \mathrm{~s}$ & $1.07 \mathrm{~s}$ & $1.71 \mathrm{~s}$ & $1.75 \mathrm{~s}$ & $1.69 \mathrm{~s}$ & $1.69 \mathrm{~s}$ & $1.69 \mathrm{~m}$ & $1.58 \mathrm{~s}$ & $1.65 \mathrm{~s}$ \\
\hline 29 & $1.38 \mathrm{~s}$ & $1.43 \mathrm{~s}$ & $1.36 \mathrm{~s}$ & $1.82 \mathrm{~s}$ & $1.37 \mathrm{~s}$ & $1.40 \mathrm{~s}$ & $1.36 \mathrm{~s}$ & $1.38 \mathrm{~s}$ & $1.38 \mathrm{~m}$ & $1.20 \mathrm{~s}$ & $1.18 \mathrm{~s}$ \\
\hline 30 & $\begin{array}{l}1.04 \mathrm{~d} \\
(6.5)\end{array}$ & $\begin{array}{l}1.10 \mathrm{~d} \\
(6.5)\end{array}$ & $\begin{array}{l}1.05 \mathrm{~d} \\
(6.8)\end{array}$ & $\begin{array}{l}1.08 \mathrm{~d} \\
(6.0)\end{array}$ & $\begin{array}{l}0.97 \mathrm{~d} \\
(7.0)\end{array}$ & $\begin{array}{l}0.98 \mathrm{~d} \\
(7.6)\end{array}$ & $\begin{array}{l}0.97 \mathrm{~d} \\
(6.8)\end{array}$ & $\begin{array}{l}0.98 \mathrm{~d} \\
(6.8)\end{array}$ & $\begin{array}{l}0.98 \mathrm{~d} \\
(7.2)\end{array}$ & $0.95 \mathrm{~s}$ & $1.10 \mathrm{~s}$ \\
\hline $3-0-$ & GlcA & GIcA & Xyl & Xyl & Xyl & Ara & Xyl & Xyl & Xyl & GlcA & GlcA \\
\hline 1 & $\begin{array}{l}4.89 d \\
(8.0)\end{array}$ & $\begin{array}{l}4.98 d \\
(5.5)\end{array}$ & $\begin{array}{l}4.88 \mathrm{~d} \\
(6.8)\end{array}$ & $\begin{array}{l}4.80 \mathrm{~d} \\
(6.4)\end{array}$ & $\begin{array}{l}4.90 \mathrm{~d} \\
(8.0)\end{array}$ & $\begin{array}{l}5.07 \mathrm{~d} \\
(7.2)\end{array}$ & $\begin{array}{l}4.95 d \\
(7.2)\end{array}$ & $\begin{array}{l}4.96 \mathrm{~d} \\
(6.0)\end{array}$ & $\begin{array}{l}4.87 d \\
(6.8)\end{array}$ & $\begin{array}{l}4.82 \mathrm{~d} \\
(6.0)\end{array}$ & $\begin{array}{l}5.00 \mathrm{~d} \\
(7.0)\end{array}$ \\
\hline 2 & $4.01 \mathrm{~m}$ & $4.05 \mathrm{~m}$ & $4.05 \mathrm{~m}$ & $4.19 \mathrm{~m}$ & $3.97 \mathrm{~m}$ & $4.20 \mathrm{~m}$ & $5.03 \mathrm{~m}$ & $4.09 \mathrm{~m}$ & $4.08 \mathrm{~m}$ & $3.97 \mathrm{~m}$ & $4.03 \mathrm{~m}$ \\
\hline 3 & $4.27 \mathrm{~m}$ & $4.23 \mathrm{~m}$ & $3.84 \mathrm{~m}$ & $4.26 \mathrm{~m}$ & $4.12 \mathrm{~m}$ & $\begin{array}{l}5.55 t \\
(8.4)\end{array}$ & $4.41 \mathrm{~m}$ & $3.85 \mathrm{~m}$ & $3.84 \mathrm{~m}$ & $4.30 \mathrm{~m}$ & $4.27 \mathrm{~m}$ \\
\hline 4 & $4.18 \mathrm{~m}$ & $4.17 \mathrm{~m}$ & $4.32 \mathrm{~m}$ & $4.21 \mathrm{~m}$ & $4.03 \mathrm{~m}$ & $4.18 \mathrm{~m}$ & $4.15 \mathrm{~m}$ & $4.19 \mathrm{~m}$ & $4.23 \mathrm{~m}$ & $4.20 \mathrm{~m}$ & $4.22 \mathrm{~m}$ \\
\hline 5 & $4.46 \mathrm{~m}$ & $4.52 \mathrm{~m}$ & $\begin{array}{l}3.68 \mathrm{~m} \\
4.28 \mathrm{~m}\end{array}$ & $\begin{array}{l}3.65 \mathrm{t} \\
(11.6) ; \\
4.29 \mathrm{dd} \\
(11.6,5.2)\end{array}$ & $\begin{array}{l}3.78 \mathrm{t} \\
(10.5) \\
4.37 \mathrm{~d} \\
(10.5)\end{array}$ & $\begin{array}{l}3.99 \mathrm{~m} \\
4.18 \mathrm{~m}\end{array}$ & $\begin{array}{l}3.68 \mathrm{~m} \\
4.30 \mathrm{~m}\end{array}$ & $\begin{array}{l}3.74 \mathrm{~m} \\
4.32 \mathrm{~m}\end{array}$ & $\begin{array}{l}3.71 \mathrm{~m} \\
4.32 \mathrm{~m}\end{array}$ & $4.44 \mathrm{~m}$ & $4.47 \mathrm{~m}$ \\
\hline $\begin{array}{l}\text { Inter- } \\
\text { mediate } \\
\text { sugar }\end{array}$ & & & Glc & & & & & Glc & Glc & & \\
\hline 1 & & & $\begin{array}{l}5.80 \mathrm{~d} \\
(7.2)\end{array}$ & & & & & $\begin{array}{l}5.44 d \\
(7.6)\end{array}$ & $\begin{array}{l}5.49 d \\
(8.0)\end{array}$ & & \\
\hline 2 & & & $4.38 \mathrm{~m}$ & & & & & $4.14 \mathrm{~m}$ & $4.17 \mathrm{~m}$ & & \\
\hline 3 & & & $4.42 \mathrm{~m}$ & & & & & $3.85 \mathrm{~m}$ & $4.28 \mathrm{~m}$ & & \\
\hline 4 & & & $4.73 \mathrm{~m}$ & & & & & $4.11 \mathrm{~m}$ & $4.37 \mathrm{~m}$ & & \\
\hline 5 & & & $4.08 \mathrm{~m}$ & & & & & $4.03 \mathrm{~m}$ & $4.25 \mathrm{~m}$ & & \\
\hline 6 & & & $\begin{array}{l}4.29 \mathrm{~m} \\
4.45 \mathrm{~m}\end{array}$ & & & & & $\begin{array}{l}3.72 \mathrm{~m} \\
4.54 \mathrm{~m}\end{array}$ & $\begin{array}{l}4.39 \mathrm{~m} \\
4.43 \mathrm{~m}\end{array}$ & & \\
\hline Terminal & & & Rha & Glc & & & & Glc & Ara & & \\
\hline 1 & & & 6.38 brs & $\begin{array}{l}5.33 d \\
(7.6)\end{array}$ & & & & $\begin{array}{l}5.38 d \\
(7.6)\end{array}$ & $\begin{array}{l}5.50 \mathrm{~d} \\
(8.0)\end{array}$ & & \\
\hline 2 & & & $4.69 \mathrm{~m}$ & $4.09 \mathrm{~m}$ & & & & $4.19 \mathrm{~m}$ & $4.20 \mathrm{~m}^{b}$ & & \\
\hline 3 & & & $4.09 \mathrm{~m}$ & $3.90 \mathrm{~m}$ & & & & $3.94 \mathrm{~m}$ & $4.10 \mathrm{~m}$ & & cont. \\
\hline
\end{tabular}


Table 1 Continued

\begin{tabular}{|c|c|c|c|c|c|c|c|c|c|c|c|}
\hline Pos. & 1 & 2 & 3 & 4 & 5 & 6 & 7 & 8 & 9 & 10 & 11 \\
\hline 4 & & & $4.26 \mathrm{~m}$ & $4.14 \mathrm{~m}$ & & & & $4.19 \mathrm{~m}$ & $4.12 \mathrm{~m}^{\mathrm{c}}$ & & \\
\hline 5 & & & $5.00 \mathrm{~m}$ & $4.26 \mathrm{~m}$ & & & & $4.05 \mathrm{~m}^{a}$ & $\begin{array}{l}3.73 \mathrm{~m} \\
4.32 \mathrm{~m}\end{array}$ & & \\
\hline 6 & & & $\begin{array}{l}1.76 \mathrm{~d} \\
(6.0)\end{array}$ & $\begin{array}{l}3.65 \mathrm{t} \\
(11.6) ; \\
4.29 \mathrm{dd} \\
(11.6,5.2)\end{array}$ & & & & $\begin{array}{l}4.42 \mathrm{~m} \\
4.56 \mathrm{~m}\end{array}$ & & & \\
\hline 28-0- & Glc & & Glc & & Glc & Glc & Glc & Glc & Glc & Glc & \\
\hline 1 & $\begin{array}{l}6.21 \mathrm{~d} \\
(8.5)\end{array}$ & & $\begin{array}{l}6.29 \mathrm{~d} \\
(8.0)\end{array}$ & & $\begin{array}{l}6.31 \mathrm{~d} \\
(8.0)\end{array}$ & $\begin{array}{l}6.32 \mathrm{~d} \\
(8.0)\end{array}$ & $\begin{array}{l}6.32 \mathrm{~d} \\
(8.0)\end{array}$ & $\begin{array}{l}6.35 \mathrm{~d} \\
(8.0)\end{array}$ & $\begin{array}{l}6.35 \mathrm{~d} \\
(8.0)\end{array}$ & $\begin{array}{l}6.33 \mathrm{~d} \\
(8.0)\end{array}$ & \\
\hline 2 & $4.20 \mathrm{~m}$ & & $4.24 \mathrm{~m}$ & & $4.22 \mathrm{~m}$ & $4.09 \mathrm{~m}$ & $4.27 \mathrm{~m}$ & $4.20 \mathrm{~m}$ & $4.20 \mathrm{~m}^{b}$ & $4.19 \mathrm{~m}$ & \\
\hline 3 & $4.02 \mathrm{~m}$ & & $4.39 \mathrm{~m}$ & & $4.04 \mathrm{~m}$ & $4.06 \mathrm{~m}$ & $4.05 \mathrm{~m}$ & $4.39 \mathrm{~m}$ & $4.38 \mathrm{~m}$ & $4.21 \mathrm{~m}$ & \\
\hline 4 & $4.37 \mathrm{~m}$ & & $4.32 \mathrm{~m}$ & & $4.19 \mathrm{~m}$ & $4.32 \mathrm{~m}$ & $4.38 \mathrm{~m}$ & $4.23 \mathrm{~m}$ & $4.12 \mathrm{~m}^{\mathrm{c}}$ & $4.37 \mathrm{~m}$ & \\
\hline 5 & $4.26 \mathrm{~m}$ & & $4.06 \mathrm{~m}$ & & $4.29 \mathrm{~m}$ & $4.28 \mathrm{~m}$ & $4.29 \mathrm{~m}$ & $4.05 \mathrm{~m}^{a}$ & $4.03 \mathrm{~m}$ & $4.27 \mathrm{~m}$ & \\
\hline 6 & $\begin{array}{l}4.36 \mathrm{~m} \\
4.44 \mathrm{~m}\end{array}$ & & $\begin{array}{l}4.37 \mathrm{~m} \\
4.45 \mathrm{~m}\end{array}$ & & $\begin{array}{l}4.37 \mathrm{~m} \\
4.42 \mathrm{~m}\end{array}$ & $\begin{array}{l}4.37 \mathrm{~m} \\
4.44 \mathrm{~m}\end{array}$ & $\begin{array}{l}4.38 \mathrm{~m} \\
4.42 \mathrm{~m}\end{array}$ & $\begin{array}{l}4.34 \mathrm{~m} \\
4.42 \mathrm{~m}\end{array}$ & $\begin{array}{l}4.39 \mathrm{~m} \\
4.43 \mathrm{~m}\end{array}$ & $\begin{array}{l}4.37 \mathrm{~m} \\
4.42 \mathrm{~m}\end{array}$ & \\
\hline
\end{tabular}

a, b, c The signals under the same superscript were overlapped

\section{Results and Discussion}

$\nabla$

Ilexpublesnin C (1) was assigned the molecular formula $\mathrm{C}_{42} \mathrm{H}_{64} \mathrm{O}_{16}$, determined on the basis of its positive HRESIMS [M + $\left.\mathrm{NH}_{4}\right]^{+}$ion peak at $m / z 842.45015$ (calcd. 842.45326). The IR spectrum showed absorption bands for hydroxyl $\left(3423 \mathrm{~cm}^{-1}\right)$, methyl $\left(2933 \mathrm{~cm}^{-1}\right)$, carbonyl $\left(1719 \mathrm{~cm}^{-1}\right)$, and olefinic $\left(1611 \mathrm{~cm}^{-1}\right)$ groups. Acid hydrolysis of $\mathbf{1}$ afforded sugar components as D-glucose and D-glucuronic acid identified by TLC and GC analyses, which was also verified by comparing ${ }^{13} \mathrm{C}$ NMR data of chikusetusaponin IVa: 107.2, 75.5, 78.1, 73.4, 77.8, 172.8 [20]. The ${ }^{1} \mathrm{H}$ and ${ }^{13} \mathrm{C}$ NMR data ( Tables 1 and 2 ) assigned by HSQC and HMBC experiments revealed five singlets for a tertiary methyl at $\delta_{\mathrm{H}} 0.72$, $1.06,1.38,1.54,1.65$, one doublet at $\delta_{\mathrm{H}} 1.04(\mathrm{~d}, J=6.5 \mathrm{~Hz})$, one olefinic linkage $\left(\delta_{\mathrm{H}} 5.49, \delta_{\mathrm{C}} 128.2, \mathrm{C}-12 ; \delta_{\mathrm{C}} 139.3, \mathrm{C}-13\right)$, two carboxyls ( $\delta_{\mathrm{C}} 177.0$, COOR-28; 175.1, COOH-GlcA-6), one low-field signal for an aldehyde group $\left(\delta_{\mathrm{H}} 10.32, \delta_{\mathrm{C}} 206.2, \mathrm{CHO}-24\right)$, and two sugar components $\left(\delta_{\mathrm{H}} 4.89, \delta_{\mathrm{C}} 106.7, \mathrm{CH}-\mathrm{GlcA}-1 ; \delta_{\mathrm{H}} 6.21, \delta_{\mathrm{C}}\right.$ 95.7, CH-Glc-1) in $\mathbf{1}$.

Analysis of the above data suggested 1 to be a hydroxylated ursane triterpene saponin with an aldehyde substitution. Detailed HMBC correlations, including from $\mathrm{H}-12$ to $\mathrm{C}-11, \mathrm{C}-14$, and $\mathrm{C}-18$, from $\mathrm{CH}_{3}-24$ to aldehyde carbon $\left(\delta_{\mathrm{C}} 206.2\right), \mathrm{C}-3$, and $\mathrm{C}-5$, from $\mathrm{CH}_{3}-29$ to $\mathrm{C}-18$ and $\mathrm{C}-20$, and from $\mathrm{CH}_{3}-30$ to $\mathrm{C}-19$ and $\mathrm{C}-20$, supported this assignment, and this analysis further indicated the structure of 1 to closely resemble ilexpublesnin A [21] except for the main difference at the sugar moiety. $\mathrm{HMBC}$ correlations ( Fig. 2) from GlcA-H-1 $\left(\delta_{\mathrm{H}} 4.89, \mathrm{~d}, J=8.0 \mathrm{~Hz}\right)$ to $\mathrm{C}-3\left(\delta_{\mathrm{C}} 86.9\right)$ and from Glc-H-1 $\left(\delta_{\mathrm{H}} 6.21, \mathrm{~d}, J=8.5 \mathrm{~Hz}\right)$ to $\mathrm{C}-28\left(\delta_{\mathrm{C}} 177.0\right)$ suggested the linkages of a 3-O-, 28-O-diglycosylation. After the construction of its planar structure, the stereochemistry was revealed by analysis of NOESY correlations ( $\odot$ Fig. 2). In the NOESY spectrum, cross-peaks of $\mathrm{CH}_{3}-29 / \mathrm{CH}_{3}-26, \mathrm{CH}_{3}-27 / \mathrm{CH}_{3}-30$ suggested an $\alpha$ configuration of $19-\mathrm{OH}$ and $\mathrm{CH}_{3}-30$, and correlations between $\mathrm{H}-24\left(\delta_{\mathrm{H}} 10.32, \mathrm{~s}\right)$ and $\mathrm{CH}_{3}-25\left(\delta_{\mathrm{H}} 0.72, \mathrm{~s}\right)$ showed that they were on the same face, confirming the $\beta$ configuration of the 24 -aldehyde. Based on the above analysis, compound 1 was elucidated as 3-0- $\beta$-D-glucuronide-3 $\beta, 19 \alpha$-dihydroxyurs-24-oxo12-en-28-oic- 0 - $\beta$-D-glucopyranosyl ester.

Ilexpublesnin D (2) was assigned the molecular formula $\mathrm{C}_{36} \mathrm{H}_{54} \mathrm{O}_{11}$ determined by the HRESIMS data. The IR, ${ }^{1} \mathrm{H}$ and ${ }^{13} \mathrm{C}$
NMR data ( Tables 1 and 2) indicated that compound 2 possessed a similar structure to 1, except for the disappearance of a glucosylation site at $\mathrm{C}-28$. The preliminary deduction was supported by comparing its ${ }^{1} \mathrm{H}$ and ${ }^{13} \mathrm{C}$ data with those of 1 and confirmed by HMBC correlations. Therefore, compound 2 was elucidated as 3-O- $\beta$-D-glucuronide-3 $\beta, 19 \alpha$-dihydroxyurs-24-oxo-12en-28-oic acid.

Analysis of the HRESIMS data $\left(\left[\mathrm{M}+\mathrm{NH}_{4}\right]^{+}\right.$at $\mathrm{m} / \mathrm{z} 1092.59670$, calcd. 1092.59490) of ilexpublesnin E (3) determined its molecular formula to be $\mathrm{C}_{53} \mathrm{H}_{86} \mathrm{O}_{22}$. Its IR spectrum showed absorption bands for hydroxyl, methyl, carbonyl, and olefinic functional groups. Acid hydrolysis of $\mathbf{3}$ afforded sugar components of D-glucose, D-xylose, and L-rhamnose identified by TLC and GC analyses. The ${ }^{1} \mathrm{H}$ and ${ }^{13} \mathrm{C}$ NMR data (Tables 1 and 2 ) revealed 3 to be a hydroxylated ursane triterpene saponin, as $\mathbf{1}$ and $\mathbf{2}$. A comprehensive analysis and comparison of its ${ }^{1} \mathrm{H}$ and ${ }^{13} \mathrm{C}$ NMR data with those of ilexoside II [22] implied that they were highly similar in structure, with the exception of an additional sugar unit in $\mathbf{3}$. HMBC correlations from the inner-xyl-H-1 $\left(\delta_{\mathrm{H}} 4.88, \mathrm{~d}, J=6.8 \mathrm{~Hz}\right)$ to $\mathrm{C}-3\left(\delta_{\mathrm{C}} 89.6\right)$, and from Glc-H-1 $\left(\delta_{\mathrm{H}} 6.29, \mathrm{~d}, J=8.0 \mathrm{~Hz}\right)$ to $\mathrm{C}-28$ $\left(\delta_{\mathrm{C}} 176.9\right)$ determined its glycosylation sites at 3-0- and 28-0-. Furthermore, HMBC correlations from the intermediate-glc- $\mathrm{H}-1$ $\left(\delta_{\mathrm{H}} 5.80, \mathrm{~d}, J=7.2 \mathrm{~Hz}\right)$ to inner-xyl-C-2 $\left(\delta_{\mathrm{C}} 79.4\right)$ and from terminal-rha-H-1 $\left(\delta_{\mathrm{H}} 6.38\right.$, br s) to intermediate-glc-C-2 $\left(\delta_{\mathrm{C}} 79.3\right)$ established all the linkages of the sugar moieties. Eventually, 3 was elucidated as 3-O-[ $\alpha$-L-rhamnopyranosyl-( $1 \rightarrow 2)-\beta$-D-glucopyranosyl-( $1 \rightarrow 2)$ - $\beta$-D-xylopyranosyl]-3 $\beta, 19 \alpha$-dihydroxyurs-12-en28-oic- $O$ - $\beta$-D-glucopyranosyl ester.

Ilexpublesnin $\mathrm{F}(4)$ was obtained as white amorphous powder with a molecular formula of $\mathrm{C}_{41} \mathrm{H}_{64} \mathrm{O}_{12}$ determined by the HRESIMS data. Overview of its IR, ${ }^{1} \mathrm{H}$ and ${ }^{13} \mathrm{C}$ NMR data ( Tables 1 and 2) implied it to be a hydroxylated ursane triterpene saponin. A careful comparison of its ${ }^{1} \mathrm{H}$ and ${ }^{13} \mathrm{C}$ NMR data with those of pubescenoside $C$ [23] revealed their structural similarity, except for the difference of no sugar moiety being present at the C-28 carboxyl in 4. All chemical and spectroscopic methods, including acid hydrolysis and HMBC experiments, established the glycosylation sites and all the linkages between sugars and aglycon. Therefore, 4 was elucidated as 3-O-[ $\beta$-D-glucopyranosyl- $(1 \rightarrow 2)]-\beta$-Dxylopyranosyl-3 $\beta$-hydroxyurs-12,18-dien-28-oic acid. 
Table $2{ }^{13} \mathrm{C}$ NMR data (in pyridine- $d_{5}$ ) of 1, 2, 5, 10, 11 (125 MHz), 3, 4, and 6-9 (100 MHz), $\delta$ in ppm.

\begin{tabular}{|c|c|c|c|c|c|c|c|c|c|c|c|}
\hline Position & 1 & 2 & 3 & 4 & 5 & 6 & 7 & 8 & 9 & 10 & 11 \\
\hline 1 & 37.9 & 37.9 & 38.8 & 39.2 & 38.6 & 38.7 & 38.8 & 38.8 & 38.9 & 37.6 & 37.6 \\
\hline 2 & 27.0 & 27.0 & 26.6 & 26.7 & 26.9 & 26.8 & 26.6 & 26.8 & 26.8 & 27.1 & 27.0 \\
\hline 3 & 86.9 & 87.0 & 89.6 & 88.8 & 88.9 & 88.4 & 89.6 & 88.9 & 88.7 & 87.0 & 87.0 \\
\hline 4 & 53.6 & 53.6 & 39.6 & 39.5 & 44.3 & 39.4 & 39.6 & 39.6 & 39.6 & 53.5 & 53.6 \\
\hline 5 & 57.6 & 57.6 & 56.0 & 56.0 & 56.3 & 55.7 & 55.9 & 55.9 & 55.9 & 57.5 & 57.5 \\
\hline 6 & 19.3 & 19.2 & 18.7 & 18.3 & 19.0 & 18.6 & 18.6 & 18.7 & 18.7 & 19.3 & 19.2 \\
\hline 7 & 33.4 & 33.4 & 33.5 & 35.6 & 33.7 & 33.4 & 33.5 & 33.5 & 33.5 & 33.0 & 33.1 \\
\hline 8 & 40.2 & 40.1 & 40.5 & 39.2 & 40.4 & 40.4 & 40.4 & 40.4 & 40.4 & 39.8 & 39.7 \\
\hline 9 & 46.4 & 46.4 & 47.7 & 48.1 & 47.6 & 47.7 & 47.7 & 47.7 & 47.8 & 47.0 & 47.0 \\
\hline 10 & 36.8 & 36.8 & 37.0 & 36.7 & 36.7 & 37.0 & 37.0 & 37.0 & 37.0 & 37.0 & 37.0 \\
\hline 11 & 24.6 & 24.4 & 24.3 & 23.4 & 24.7 & 24.7 & 24.7 & 24.7 & 24.7 & 24.6 & 24.5 \\
\hline 12 & 128.2 & 127.8 & 128.3 & 126.3 & 127.6 & 127.7 & 127.6 & 127.6 & 127.7 & 123.5 & 123.5 \\
\hline 13 & 139.3 & 140.0 & 139.2 & 139.5 & 138.8 & 138.8 & 138.8 & 138.8 & 138.2 & 144.2 & 144.9 \\
\hline 14 & 42.1 & 42.1 & 42.1 & 44.7 & 42.1 & 42.2 & 42.1 & 42.1 & 42.1 & 42.0 & 42.1 \\
\hline 15 & 29.2 & 29.2 & 29.2 & 28.8 & 29.2 & 29.2 & 29.2 & 29.2 & 29.2 & 28.4 & 28.3 \\
\hline 16 & 24.8 & 24.7 & 26.6 & 34.8 & 26.8 & 26.6 & 26.6 & 26.8 & 26.6 & 28.9 & 29.1 \\
\hline 17 & 48.6 & 48.2 & 48.6 & 50.2 & 48.3 & 47.9 & 48.3 & 48.3 & 48.3 & 46.4 & 46.0 \\
\hline 18 & 54.4 & 54.6 & 54.4 & 135.0 & 27.2 & 47.2 & 47.2 & 47.2 & 47.2 & 44.6 & 44.8 \\
\hline 19 & 72.6 & 72.7 & 72.6 & 135.9 & 73.4 & 73.4 & 73.4 & 73.4 & 73.4 & 81.0 & 81.2 \\
\hline 20 & 42.1 & 42.4 & 42.1 & 37.4 & 42.8 & 42.8 & 42.8 & 42.9 & 42.8 & 35.5 & 35.7 \\
\hline 21 & 26.0 & 27.1 & 27.0 & 29.2 & 24.2 & 24.1 & 24.0 & 24.0 & 24.0 & 28.9 & 29.2 \\
\hline 22 & 37.6 & 38.5 & 36.9 & 34.8 & 31.8 & 31.9 & 31.8 & 31.9 & 31.9 & 33.1 & 33.6 \\
\hline 23 & 21.4 & 21.5 & 28.4 & 28.1 & 23.4 & 28.3 & 28.3 & 28.2 & 28.2 & 21.3 & 21.5 \\
\hline 24 & 206.2 & 206.2 & 16.7 & 16.7 & 63.3 & 16.0 & 17.4 & 16.8 & 16.8 & 206.4 & 205.9 \\
\hline 25 & 16.2 & 16.1 & 15.6 & 16.1 & 15.4 & 15.6 & 15.6 & 15.7 & 15.7 & 15.9 & 15.9 \\
\hline 26 & 17.2 & 17.0 & 17.4 & 18.0 & 17.4 & 17.5 & 16.9 & 17.5 & 17.5 & 17.4 & 17.2 \\
\hline 27 & 24.5 & 24.6 & 24.0 & 22.2 & 24.2 & 24.4 & 24.3 & 24.3 & 24.3 & 24.8 & 24.8 \\
\hline 28 & 177.0 & 180.6 & 176.9 & 178.9 & 177.0 & 177.1 & 177.1 & 177.0 & 177.0 & 177.2 & 180.8 \\
\hline 29 & 26.7 & 26.9 & 26.8 & 20.2 & 29.7 & 29.7 & 29.7 & 29.7 & 29.7 & 28.8 & 28.8 \\
\hline 30 & 16.7 & 16.8 & 16.6 & 20.5 & 16.0 & 17.0 & 16.0 & 16.0 & 16.0 & 24.6 & 24.7 \\
\hline 3-O- & GlcA & GlcA & Xyl & Xyl & Xyl & Ara & Xyl & Xyl & Xyl & GlcA & GlcA \\
\hline 1 & 106.7 & 107.4 & 105.8 & 105.7 & 106.7 & 106.3 & 104.8 & 105.0 & 105.2 & 106.5 & 107.3 \\
\hline 2 & 75.1 & 75.2 & 79.4 & 82.7 & 75.3 & 74.1 & 80.2 & 82.1 & 82.6 & 75.0 & 75.2 \\
\hline 3 & 78.2 & 78.0 & 77.7 & 77.8 & 78.5 & 84.0 & 77.4 & 77.8 & 77.8 & 76.8 & 78.0 \\
\hline 4 & 73.4 & 73.4 & 71.2 & 71.5 & $71.2^{c}$ & 69.9 & 70.8 & 71.1 & 71.6 & 73.4 & 73.4 \\
\hline 5 & 76.8 & 76.8 & 66.6 & 66.6 & 67.2 & 66.2 & 65.9 & 65.9 & 66.5 & 76.8 & 77.9 \\
\hline 6 & 175.1 & $-a$ & & & & & & & & 176.0 & $-a$ \\
\hline Intermediate & & & Glc & & & & & Glc & Glc & & \\
\hline 1 & & & 102.3 & & & & & 103.2 & 103.2 & & \\
\hline 2 & & & 79.3 & & & & & 85.4 & 84.4 & & \\
\hline 3 & & & 79.2 & & & & & 77.8 & $77.9 f$ & & \\
\hline 4 & & & 72.6 & & & & & 70.1 & 70.7 & & \\
\hline 5 & & & $79.0^{b}$ & & & & & 77.8 & $78.0^{f}$ & & \\
\hline 6 & & & 63.2 & & & & & 62.7 & 62.7 & & \\
\hline Terminal & & & Rha & Glc & & & & Glc & Ara & & \\
\hline 1 & & & 102.0 & 105.6 & & & & 106.4 & 106.5 & & \\
\hline 2 & & & 72.3 & 76.8 & & & & 76.6 & 71.1 & & \\
\hline 3 & & & 72.6 & 77.9 & & & & $79.1^{e}$ & 76.0 & & \\
\hline 4 & & & 74.3 & 70.8 & & & & 71.0 & 70.5 & & \\
\hline 5 & & & 69.5 & 78.3 & & & & 79.3 & 67.4 & & \\
\hline 6 & & & 19.0 & 62.6 & & & & 62.5 & & & \\
\hline 28-0- & Glc & & Glc & & Glc & Glc & Glc & Glc & Glc & Glc & \\
\hline 1 & 95.7 & & 95.8 & & 95.8 & 95.8 & 95.8 & 95.8 & 95.8 & 95.8 & \\
\hline 2 & 74.0 & & 74.1 & & 74.1 & 73.9 & 74.1 & 74.1 & 74.1 & 74.1 & \\
\hline 3 & 78.8 & & 78.4 & & 79.0 & 78.9 & $79.0^{d}$ & $79.0^{e}$ & 79.0 & 78.9 & \\
\hline 4 & 71.2 & & 71.2 & & $71.1^{c}$ & 71.1 & 71.1 & 71.1 & 70.7 & 71.0 & \\
\hline 5 & 79.2 & & $78.9^{b}$ & & 79.2 & 79.2 & $79.2^{d}$ & 79.3 & 79.3 & 79.3 & \\
\hline 6 & 62.2 & & 62.3 & & 62.2 & 62.2 & 62.2 & 62.2 & 62.2 & 62.0 & \\
\hline
\end{tabular}

a Signals were absent; $b, c, d, e, f$ The signals under the same superscript may be interchanged 


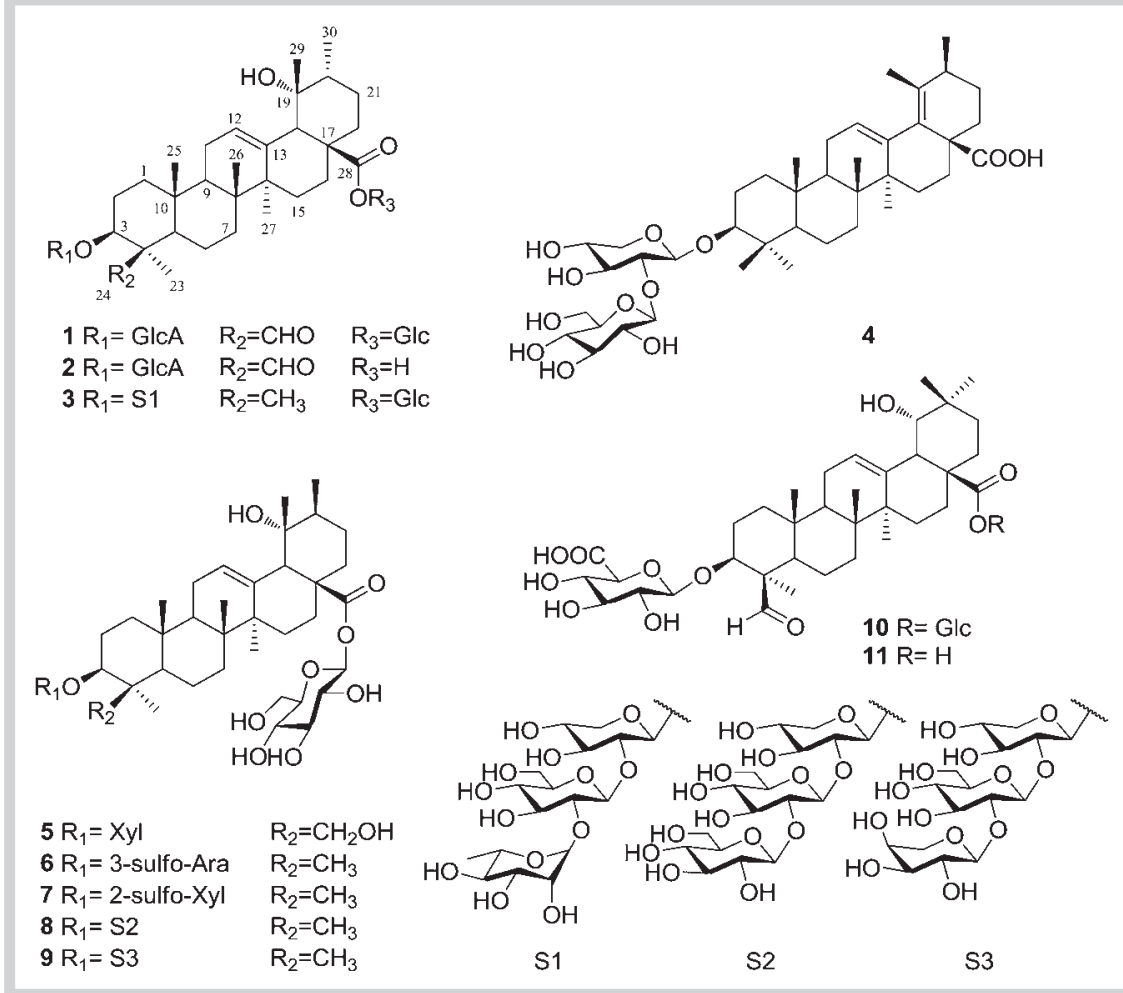

Fig. 1 Structures of compounds 1-11.
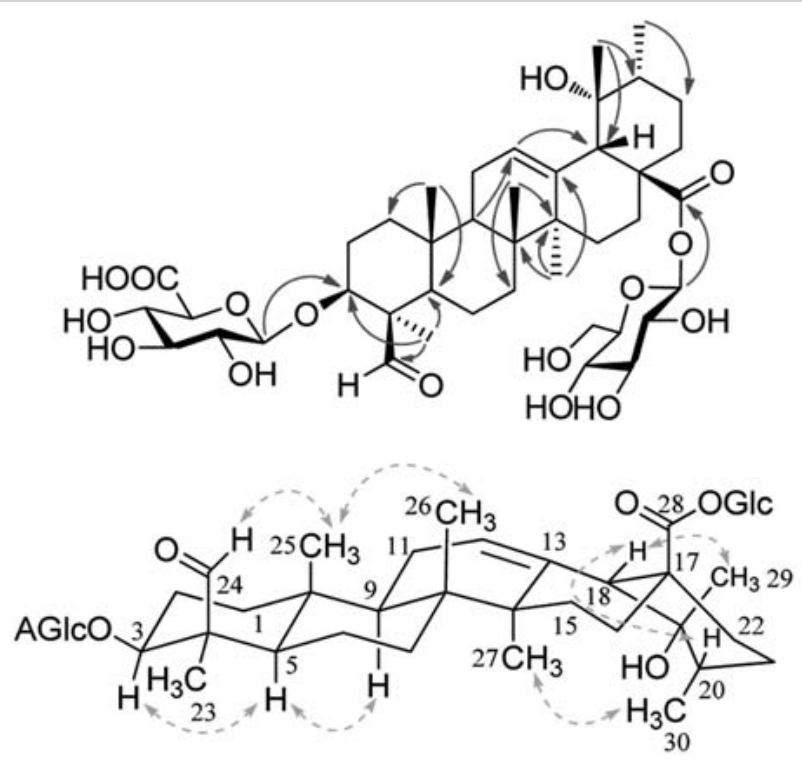

HMBC $\frown$ NOESY $\cdots$

Fig. 2 Key HMBC and NOESY correlations of $\mathbf{1}$.

Ilexpublesnin $\mathrm{G}$ (5) was given the molecular formula $\mathrm{C}_{41} \mathrm{H}_{66} \mathrm{O}_{14}$ by HRESIMS data $\left([2 \mathrm{M}+\mathrm{H}]^{+}\right.$at $\mathrm{m} / \mathrm{z}$ 1565.89765, calcd. 1565.89779). The IR spectrum showed absorption bands for hydroxyl, methyl, carbonyl, and olefinics. Acid hydrolysis of 5 afforded D-glucose and D-xylose, identified by TLC and GC analyses. The signals characteristic for an ursane triterpene saponin were observed from its ${ }^{1} \mathrm{H}$ and ${ }^{13} \mathrm{C}$ NMR data ( Tables 1 and 2 ), including five singlets for tertiary methyls, an olefinic proton at
$\delta_{\mathrm{H}} 5.48$ (br s), and two anomeric carbons as well. Detailed interpretation of its ${ }^{1} \mathrm{H}$ and ${ }^{13} \mathrm{C}$ NMR data suggested 5 to be closely related to ilexoside A [2], except for the oxidization of $24-\mathrm{CH}_{3}$ leading to 24-hydroxymethine, which was consistent with the chemical shifts of $\mathrm{C}-5\left(\delta_{\mathrm{C}} 56.3\right)$ and C-23 $\left(\delta_{\mathrm{C}} 23.4\right)$, respectively, compared to chemical shifts of $\mathrm{C}-5\left(\delta_{\mathrm{C}} 48.2\right)$ and $\mathrm{C}-24\left(\delta_{\mathrm{C}} 14.3\right)$ when hydroxylation happened at C-23 $[24,25]$. This was further confirmed by a NOESY correlation between $\mathrm{H}-24 \mathrm{a}\left(\delta_{\mathrm{H}} 4.36\right)$ and $\mathrm{CH}_{3}-25$. Finally, 5 was elucidated as $3-0-\beta$-D-xylpyranosyl$3 \beta, 19 \alpha, 24 \beta$-trihydroxyurs-12-en-28-oic-O- $\beta$-D-glucopyranosyl ester.

The molecular formula $\mathrm{C}_{41} \mathrm{H}_{66} \mathrm{O}_{16} \mathrm{~S}$ was determined for ilexpublesnin $\mathrm{H}(\mathbf{6})$ on the basis of its negative HRESIMS ([M - H $]^{-}$at $m / z$ 845.39863, calcd. 845.39988). Overall appearance of its IR spectrum and ${ }^{1} \mathrm{H},{ }^{13} \mathrm{C}$ NMR data ( Tables 1 and 2 ) displayed a high resemblance with those of ilexoside $A$ [2]. Interpretation of the spectroscopic data of 6 revealed the only difference between both compounds to be a sugar moiety. Acid hydrolysis of 6 yielded ilexgenin B [26], D-glucose, and L-arabinose identified by TLC and GC analyses. Comparison of the ${ }^{13} \mathrm{C}$ NMR data with those of ziyu-glycoside I [27] revealed sulfonylation on the hydroxyl attaching to Ara-C-3, which was supported by HMBC correlations from Ara-H-3 $\left(\delta_{\mathrm{H}} 5.50\right)$ to Ara-C-2 $\left(\delta_{\mathrm{C}} 74.1\right)$ and Ara-C-4 $\left(\delta_{\mathrm{C}} 69.9\right)$. The same analysis of the HMBC correlations from Ara$\mathrm{H}-1\left(\delta_{\mathrm{H}} 5.07\right)$ to $\mathrm{C}-3\left(\delta_{\mathrm{C}} 88.4\right)$, and from Glc-H-1 $\left(\delta_{\mathrm{H}} 6.32\right)$ to $\mathrm{C}-28$ $\left(\delta_{\mathrm{C}} 177.1\right)$ suggested 6 to be a 3-O-, 28-O-diglycoside of ilexgenin B. Subsequently, 6 was elucidated as $3-0-\alpha$-L-(3'-sulfonyl)-arabinpyranosyl ilexgenin B 28-O- $\beta$-D-glucopyranosyl ester.

The molecular formula $\mathrm{C}_{41} \mathrm{H}_{66} \mathrm{O}_{16} \mathrm{~S}$ for ilexpublesnin I (7) was given by HRESIMS data. The IR, ${ }^{1} \mathrm{H}$ and ${ }^{13} \mathrm{C}$ NMR data ( $\odot$ Tables 1 and 2 ) exhibited a high similarity with those of $\mathbf{6}$, suggesting related structures. Acid hydrolysis of 7 gave ilexgenin B, D-glucose, and D-xylose after TLC and GC analyses. Comparison of the ${ }^{13} \mathrm{C}$ NMR data with those of ilexoside A [2] revealed sulfonylation on 
the hydroxyl attaching to Xyl-C-2 compared to 7. HMBC correlations from Xyl-H-2 $\left(\delta_{\mathrm{H}} 5.03, \mathrm{~m}\right)$ to Xyl-C-1 $\left(\delta_{\mathrm{C}} 104.8\right)$ and Xyl-C-3 $\left(\delta_{\mathrm{C}} 77.4\right)$ confirmed these assignments. Eventually, an extensive analysis of the HMBC correlations of 7 was applied to establish its structure as 3-O- $\beta$-D-(2'-sulfonyl)-xylpyranosyl ilexgenin $\mathrm{B}$ $28-0$ - $\beta$-D-glucopyranosyl ester.

Ilexpublesnin J (8) was deduced to have the molecular formula $\mathrm{C}_{53} \mathrm{H}_{86} \mathrm{O}_{23}$ by HRESIMS ([M $\left.+\mathrm{NH}_{4}\right]^{+}$at $\mathrm{m} / z$ 1108.58661, calcd. 1108.58981). Acid hydrolysis of 8 afforded ilexgenin B, D-glucose, and D-xylose after identification by TLC and GC analyses. The ${ }^{1} \mathrm{H}$ and ${ }^{13} \mathrm{C}$ NMR data ( Tables 1 and 2 ) assigned by various NMR experiments exhibited similarity with those of ilexsaponin $B_{3}[28]$, indicating their similar structures. Comparison of their ${ }^{1} \mathrm{H}$ and ${ }^{13} \mathrm{C}$ NMR data revealed that 8 had one additional sugar unit, supported by HMBC correlations from inner-xyl-H-1 $\left(\delta_{\mathrm{H}} 4.96\right)$ to $\mathrm{C}-3$ $\left(\delta_{\mathrm{C}} 88.9\right)$ and from Glc-H-1 $\left(\delta_{\mathrm{H}} 6.35\right)$ to $\mathrm{C}-28\left(\delta_{\mathrm{C}} 177.0\right)$. This determined for 8 a 3-0-, 28-0-disaccharide structure. The positions and sequence of the rest of the sugar moieties were defined by HMBC correlations from intermediate-glc-H-1 $\left(\delta_{\mathrm{H}} 5.44\right)$ to inner-xyl-C-2 $\left(\delta_{\mathrm{C}} 82.1\right)$ and from terminal-glc-H-1 $\left(\delta_{\mathrm{H}} 5.38\right)$ to intermediate-glc-C-2 ( $\left.\delta_{\mathrm{C}} 85.4\right)$. Thus, 8 was unambiguously elucidated as $3-O$-[ $\beta$-D-glucopyranosyl- $(1 \rightarrow 2)-\beta$-D-glucopyranosyl$(1 \rightarrow 2)$ ]- $\beta$-D-xylopyranosyl ilexgenin B $28-0-\beta$-D-glucopyranosyl ester.

Ilexpublesnin $\mathrm{K}(9)$ has the molecular formula $\mathrm{C}_{52} \mathrm{H}_{84} \mathrm{O}_{22}$ determined by its HRESIMS data. Comparison of its ${ }^{1} \mathrm{H}$ and ${ }^{13} \mathrm{C}$ NMR data ( Tables $\mathbf{1}$ and $\mathbf{2}$ ) with those of $\mathbf{8}$ suggested that they were closely related in structures, except for a difference in the sugar moiety. Acid hydrolysis of $\mathbf{9}$ afforded D-glucose, D-xylose, and Larabinose which were identified by TLC and GC analyses. The structure assignments and the linkages of the sugar moiety were confirmed by HMBC correlations. Accordingly, 9 was elucidated as 3 - $O$-[ $\alpha$-L-arabinpyranosyl-( $1 \rightarrow 2)-\beta$-D-glucopyranosyl- $(1 \rightarrow$ $2)]-\beta$-D-xylopyranosyl ilexgenin B $28-O-\beta$-D-glucopyranosyl ester.

Ilexpublesnin L (10) and ilexpublesnin M (11) were obtained as white amorphous powders. Their molecular formulas were determined as $\mathrm{C}_{42} \mathrm{H}_{64} \mathrm{O}_{16}$ and $\mathrm{C}_{36} \mathrm{H}_{54} \mathrm{O}_{11}$, respectively, by the HRESIMS data. The IR spectra of both compounds showed absorption bands for hydroxyl, methyl, carbonyl, and olefinics. The ${ }^{1} \mathrm{H}$ and ${ }^{13} \mathrm{C}$ NMR data ( Tables 1 and 2 ) displayed signals for six methyls, an olefinic, an aldehyde, and a sugar moiety, including glucose and glucuronic acid for $\mathbf{1 0}$ and a glucuronic acid for 11, respectively. These assignments were confirmed by acid hydrolysis and/or ${ }^{1} \mathrm{H}$ and ${ }^{13} \mathrm{C}$ NMR comparisons with reported data. Analysis of ${ }^{13} \mathrm{C}$ NMR data of their aglycones indicated that both $\mathbf{1 0}$ and $\mathbf{1 1}$ closely resembled ilexoside XXXIII [29], with an aldehyde on C24 in 10 and 11, differently from ilexoside XXXIII with the aldehyde substitution on C-23. The determination was further supported by the observation of NOESY correlations between $\mathrm{H}-24$ with $\mathrm{CH}_{3}-25$, suggesting the $\beta$ configuration of the aldehyde.

The complete structures, including all the linkages of sugars and aglycones, were established by extensive HMBC correlation analyses. Therefore, 10 and 11 were elucidated as 3- 0 - $\beta$-D-glucuronide-3 $\beta$, $19 \alpha$-dihydroxyolea-24-oxo-12-en-28-oic- $O-\beta$-D-glucopyranosyl ester and 3-O- $\beta$-D-glucuronide-3 $\beta, 19 \alpha$-dihydroxyolea-24-oxo-12-en-28-oic acid, respectively.

Additionally, ten known analogues (12-21) (see Supporting Information) were also isolated. By comparing their ${ }^{1} \mathrm{H}$ and ${ }^{13} \mathrm{C}$ NMR as well as MS data with reported values, their structures were identified as ilexoside II (12) [22], ilexsaponin $B_{1}$ (13) [28], ilexsaponin $\mathrm{B}_{3}(\mathbf{1 4})$ [28], ilexoside $\mathrm{O}$ (15) [30], ilexsaponin $\mathrm{B}_{4}(\mathbf{1 6})$
[31], ilexoside XV (17) [32], ilexsaponin C (18) [33], chikusetusaponin IVa (19) [22], oleanolic acid 28-O- $\beta$-D-glucopyranol ester (20) [34], and pubescenoside C (21) [4].

Compounds $\mathbf{1}, \mathbf{2}, \mathbf{1 0}$, and 11 are considerably special, as they contain the rare 24-aldehyde group, compared to the common 23-aldehyde- [21] or 24-COOH-substituted triterpene saponins from Ilex species.

Compounds 1-21 were evaluated for their cytotoxic activities in vitro against the human hepatoma cell lines HepG2, HLE, BEL7402, BEL-7403, BEL-7405, human breast cancer cell line MCF-7, and human cervical cancer cell line HeLa, using the MTT assay [20]. Unfortunately, no significant cytotoxic results [inhibition $(\%)<50$ ] were observed against all seven cancer cell lines at the concentration of $40 \mu \mathrm{M}$. Among these isolates, only 6 and 19 showed cytotoxicity against the MCF-7 cell line [inhibition $(\%)=33.14,34.03$, respectively], and 20 exhibited a weak cytotoxic activity on the HeLa cell line [inhibition (\%) = 36.86]. The cytotoxic effects of $\mathbf{1 9}$ and $\mathbf{2 0}$ may be due to their aglycon oleanic acid.

\section{Acknowledgments}

$\nabla$

The authors express their thanks to the analytical center of Peking University Health Science Center for measurements of MS and NMR. This work was financially supported by grants from the Natural Science Foundation of China (No. 30672608) and the scientific and technological major special project for 'Significant Creation of New Drugs' (2009ZX09311-004).

\section{Conflict of Interest}

$\nabla$

The authors of the present manuscript have declared that no competing interests exist.

\section{References}

1 Jiangsu New Medical College. Dictionary of chinese materia medica. Shanghai: Shanghai Scientific and Technological Press; 1977: 441

2 Inada A, Kobayashi M, Murata $H$. Two new triterpenoid glycosides from leaves of Ilex chinensis sims. Chem Pharm Bull 1987; 35: 841-845

3 Zhou YB, Wang JH, Li XM, Li X. A new lignan derivative from the root of Ilex pubescens. Chin Chem Lett 2007; 19: 550-552

4 Yang $X$, Ding Y, Sun ZH, Zhang DM. Studies on chemical constituents from Ilex pubescens. J Asian Nat Prod Res 2006; 8: 505-510

5 Zhou YB, Wang JH, Li XM, Fu XC, Yan Z, Cong Y, Li X. Two new 2-phenylethyl alcohol derivatives and one new lignan derivative from the root of Ilex pubescens. Helv Chim Acta 2008; 91: 1244-1250

6 Jiang ZF, Huang RX, Qin GW, Tian Y, Xu RS. Studies on chemical constituents from Ilex pubescens III. Isolation and identification of four triterpene saponins. Tradit Herb Drugs 1991; 22: 291

7 Han YN, Song JI, Rhee IK. Anticoagulant activity of ileoside D, a triterpenoid from Ilex pubescens. Arch Pharm Res 1993; 16: 209-212

8 Jiang ZH, Wang JR, Li M, Liu ZQ Chau KY, Zhao C, Liu L. Hemiterpene glucosides with anti-platelet aggregation activities from Ilex pubescens. J Nat Prod 2005; 68: 397-399

9 Qin WJ, Jiao ZY, Fan ZT, Chen BQ Lin XY, Yao JX. Studies on chemical constituents from the leaves of Ilex pubescens. Acta Pharm Sin 1980; 11 : 199

10 Zheng J, Tang L, Xian XD, Zhou SX, Shi HM, Jiang Y, Gu YQ Liu G, Tu PF. Inhibitory effect of triterpenoid saponins from the leaves of Ilex $k u$ dingcha on aggregated LDL-induced lipid deposition in macrophages. Planta Med 2009; 75: 1410-1414

11 Zheng J, Wang X, Li H, Gu YQ Tu PF, Wen Z. Improving abnormal hemorheological parameters in ApoE-/- mice by Ilex total saponins. Clin Hemorheol Microcirc 2009; 42: 29-36 
12 Tang L, Jiang Y, Chang HT, Zhao MB, Tu PF, Cui JR, Wang RQ. Triterpene saponins from the leaves of Ilex kudingcha. J Nat Prod 2005; 68: 11691174

13 Tang L, Jiang Y, Tian XM, Zhou SX, Tu PF. Triterpene saponins from the leaves of Ilex kudingcha. J Asian Nat Prod Res 2009; 11: 553-560

14 Zhou SX, Yang J, Liu F, Tu PF. Complete assignments of ${ }^{1} \mathrm{H}$ and ${ }^{13} \mathrm{C}$ NMR spectroscopic data for two new triterpenoid saponins from Ilex hainanensis. Magn Reson Chem 2007; 45: 179-181

15 Zhou SX, Yang J, Liu F, Tu PF. Triterpenoids from the leaves of Ilex hainanensis. Helv Chim Acta 2007; 90: 121-127

16 Xie GB, Lei $L D, T u P F$. Complete assignments of ${ }^{1} \mathrm{H}$ and ${ }^{13} \mathrm{C}$ NMR spectroscopic data for two new triterpenoid saponins from Ilex pernyi. Magn Reson Chem 2007; 45: 997-1000

17 Xie GB, Zheng J, Tu PF, Liu GQ. Triterpene saponins from the leaves of Ilex pernyi. Helv Chim Acta 2008; 91: 1630-1639

18 Xie GB, Zhou SX, Lu YN, Lei LD, Tu PF. Triterpenoid glycoside from the leaves of Ilex pernyi. Chem Pharm Bull 2009; 57: 520-524

19 Mosmann T. Rapid colorimetric assay for cellular growth and survival: application to proliferation and cytotoxicity assays. J Immunol Methods 1983; 65: 55-63

20 Junei K, Kumi S, Toshihiro N. Triterpenoidal saponins from Dumasia truncate. Phytochemistry 1997; 40: 1765-1767

21 Zhang CX, Lin CZ, Xiong TQ Zhu CC, Yang JY, Zhao ZX. New triterpene saponins from the root of Ilex pubescens. Fitoterapia 2010; 81: $788-792$

22 Tsutomu N, Hiroko T, Masao N. Two triterpenoid glycosides from leaves of Ilex cornuta. Phytochemistry 1982; 21: 1373-1377

23 Wang JR, Zhou H, Zhang ZH. Two new triperpene saponins from the anti-inflammatory saponin fraction of Ilex pubescens root. Chem Biodivers 2008; 5: 1369-1376
24 Mahato SB, Kundu AP. ${ }^{13} \mathrm{C}$ NMR spectra of pentacyclic triterpenoids - a compilation and some salient features. Phytochemistry 1994; 37: 1517-1575

25 Zhao YY. Medicinal chemistry of natural products, Chapter 7. Beijing: People's Medical Publishing House Press; 2005: 271-311

26 Ouyang MA, Wang HQ Liu YQ Yang CR. Triterpenoid saponins from the leaves of Ilex latifolia. Phytochemistry 1997; 45: 1501-1505

27 Itiro $Y$, Tamio S, Akio O, Isao K. Soil bacterial hydrolysis leading to genuine aglycone. III. The structures of glycosides and genuine aglycone of Sanguisorbae Radix. Chem Pharm Bull 1971; 19: 1700-1707

28 Kazuyuki H, Miyoko I, Yoko M. New triterpene saponins from Ilex pubescens. Chem Pharm Bull 1987; 35: 524-529

29 Kayoko A, Kazuko Y, Shigenhou A. Triterpenoid saponins of aquifoliaceous plants. XI. Ilexosides XLI-XLV from the leaves of Ilex rotunda Thumb. Chem Pharm Bull 1993; 41: 39-42

30 Han YN, Han BH, Baik SK. Antithrombotic activity of the triterpenoids of Ilex pubescens and the conversion method of the triterpenoids of Ilex pubescens having no antithrombotic activity into the triterpenoids having antithrombotic activity. US Patent 4987125; 1991

31 Wang CZ, Jia ZJ. Phenylpropanoid and iridoid glycosides from Pedicularis torta. Phytochemistry 1997; 45: 159-166

32 Chieko H, Miho K, Kazuko Y. Triterpenoid saponins of aquifoliaceous plants. V. Ilexosides XV-XIX from the barks of Ilex crenata Thumb. Chem Pharm Bull 1992; 40: 1990-1992

33 Feng F, Zhu MX, Xie N, Liu WY, Chen DJ, You QD. Two new triterpenoid saponins from the root of Ilex pubescens. J Asian Nat Prod Res 2009; 10: 71-75

34 Lin YP, Qiu MH, Li ZR, Gao XH. New triterpenoid glycosides from Hemsleya penxianensis var. gulinensis. Acta Bot Yunn 2003; 25: 235-240 